\title{
Measurement of vibration velocity pattern of facial surface during phonation using scanning vibrometer
}

\author{
Tatsuya Kitamura* \\ Faculty of Intelligence and Informatics, Konan University, \\ 8-9-1 Okamoto, Higashinada-ku, Kobe, 658-8501 Japan
}

(Received 3 October 2011, Accepted for publication 4 November 2011)

Keywords: Scanning laser-Doppler vibrometer, Radiation of speech sounds, Vibration velocity, Vowel, Nasal consonant PACS number: 40.70.Jt [doi:10.1250/ast.33.126]

\section{Introduction}

Speech sound is radiated not only from the mouth and nostril openings but also from the surfaces of the head and neck $[1,2]$. The vibration pattern of the head and neck surfaces or the contribution of the sound radiated from the surfaces to the overall sound, however, has been inadequately studied. One of the reasons for this is the difficulty of measuring the physical phenomenon; it is difficult to measure the vibration of the surfaces and to record the sound radiated from the surfaces separately from that radiated from the mouth and nostril openings. In this paper, we report the results of a pilot study for the measurement of the vibration velocity patterns of the facial surface during sustained phonations of a vowel and a nasal consonant using a scanning laser-Doppler vibrometer.

In their pioneering work, Kirikae et al. [3] used vibrometers to measure phonatory body vibrations. They measured vibrations caused by phonation at more than forty points on the body. Suzuki et al. [4] employed acceleration pickups and measured the acceleration of the nasal wall, the neck wall, and the cheek during speech production. Toyoda and Fujinami [5] utilized an optical fiber sensor to measure the vibration at nine different locations on the body during phonation. It is, however, undeniable that the sensors used in contact with the measurement objects affect their vibration, although the acceleration pickup used by Suzuki et al. [4] is light $(0.4 \mathrm{~g})$ and small (external diameter of $5.84 \mathrm{~mm}$ ).

Pawluczyk and Kraska [6] developed a laser-based method that can show equal-vibration-amplitude contours of objects, and measured the nodal patterns on the facial and neck surfaces during singing. Since it is a noncontact method, the vibrations were completely unaffected during the measurements. The scanning vibrometer used in this study also allows laser-based, noncontact, and multipoint measurements of the vibration of objects. Moreover, this method has the advantage of enabling the analysis of the vibration for a particular frequency band of interest.

\section{Methods}

2.1. Scanning vibrometer

Vibration velocity was obtained with a scanning laser-

*e-mail: t-kitamu@konan-u.ac.jp
Doppler vibrometer system (Polytec PSV-400-M4). The laserDoppler vibrometer is an optical transducer that senses the frequency shift of the light reflected from a vibrating surface on the basis of the Doppler effect and can determine the vibration velocity and displacement at a certain point [7]. The scanning vibrometer can scan and probe multiple points of a vibrating surface automatically.

\subsection{Participant}

A Japanese male (22 years of age) with no history of previous or current speech impairment participated in the measurements.

\subsection{Data acquisition}

Figure 1 shows the experimental setup. The scanning head of the vibrometer was mounted on a tripod, perpendicular to the floor. The participant was positioned to lie supine directly beneath the scanning head with his head stabilized by wooden blocks. He wore protective goggles to protect his eyes from the laser emission.

Data were acquired during sustained phonations of the Japanese vowel /a/ and nasal consonant /N/. The participant was asked to articulate utterances repeatedly while keeping his head immobile during the measurements. Speech sounds were recorded through a microphone (Audio-technica AT9820X).

The vibrometer can measure the vibration velocity and displacement of the direction of the laser light. In this study, we thus measured the vibration patterns of the facial surface from the frontal direction, which is perpendicular to the forehead, and from an oblique direction, which is nearly perpendicular to the left cheek and the left side of the nose of the participant.

In the experiment, scanning points on the facial surface were first determined using system control software. 75 and 49 points were set for the frontal direction and the left oblique direction to be scanned, respectively. During the measurement, the vibrometer scanned each point and determined the vibration velocity of the points. It took approximately $1 \mathrm{~s}$ to probe one point. The vibration velocity and speech sounds were measured up to $5 \mathrm{kHz}$.

Written informed consent was obtained from the participant prior to the measurement. The experimental protocol was approved by the ethical and safety committees of Konan University. 


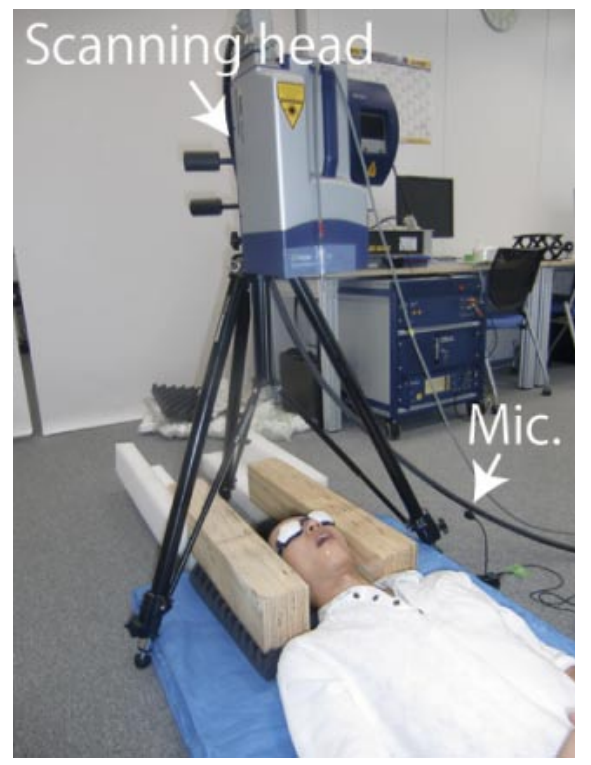

Fig. 1 Experimental setup.

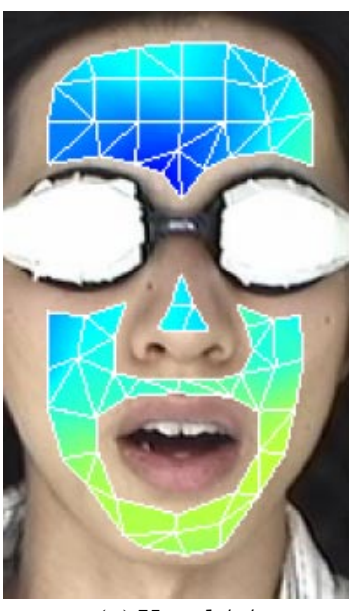

(a) Vowel /a/

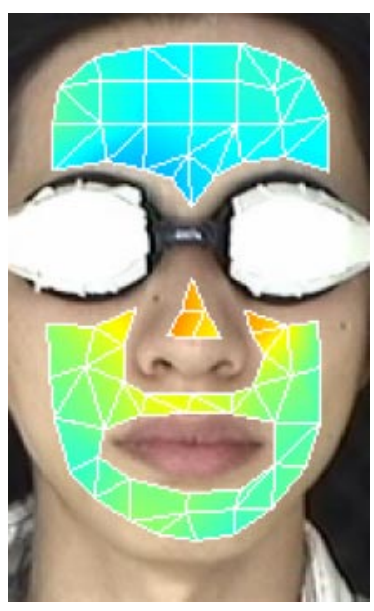

(b) Nasal consonant /N/

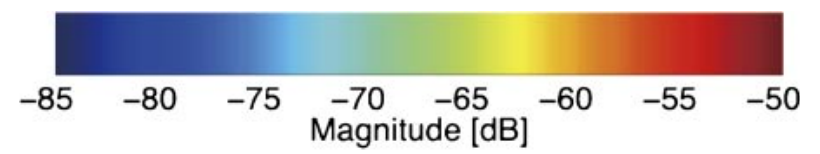

Fig. 2 Vibration velocity patterns of frontal facial surface during articulation of (a) Japanese vowel /a/ and (b) nasal consonant $/ \mathrm{N} /$. The unit is $\mathrm{m} / \mathrm{s}[\mathrm{dB}]$ and $0 \mathrm{~dB}$ is equal to $1 \mathrm{~m} / \mathrm{s}$.

\section{Results}

Figures 2(a) and 2(b) show the vibration velocities of the frontal facial surface in $\mathrm{dB}$ during sustained phonations of the vowel /a/ and nasal consonant $/ \mathrm{N} /$, respectively. The grid points in the figures are the scanning points and the values of the other points were interpolated from that of the grid points. These figures confirm that the scanning vibrometer enables us to study the vibration of the skin surface during phonation.

There were significant differences between the vibration velocity patterns for the vowel and nasal consonant. For the

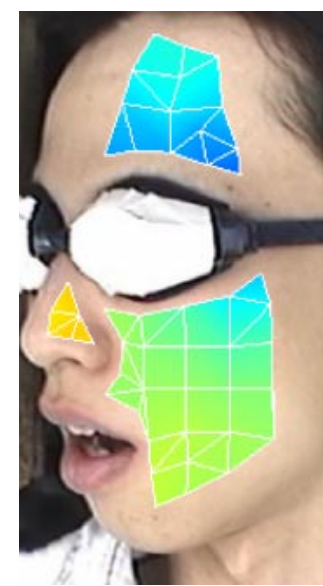

(a) Vowel /a/

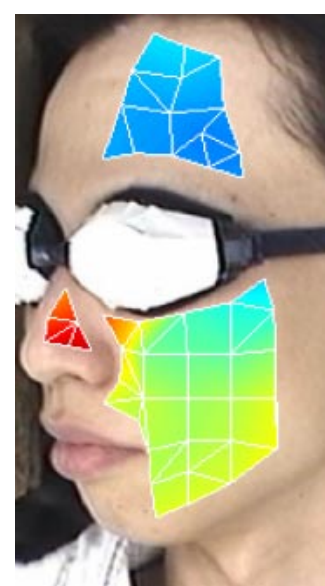

(b) Nasal consonant $/ \mathrm{N} /$

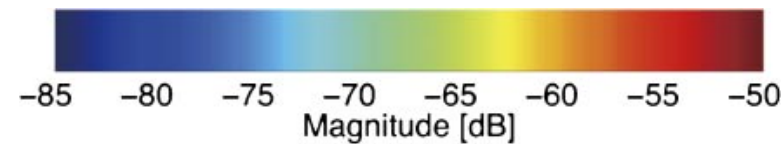

Fig. 3 Vibration velocity patterns of left facial surface during articulation of (a) Japanese vowel /a/ and (b) nasal consonant $/ \mathrm{N} /$. The unit is $\mathrm{m} / \mathrm{s}[\mathrm{dB}]$ and $0 \mathrm{~dB}$ is equal to $1 \mathrm{~m} / \mathrm{s}$.

vowel, the facial surface around the mouth opening vibrated greatly relative to the other regions. In contrast, for the nasal consonant, the facial surfaces of the nose and its vicinity vibrated strongly owing to the resonance in the nasal sinuses. The forehead surface also vibrated to some extent, possibly indicating that the frontal sinuses resonated during the production of the nasal consonant.

Figure 3 shows the vibration velocity patterns of the left facial surface for the phonemes. The vibration of the side of the nose was observed to be stronger for both phonemes than that indicated in Fig. 2. This means that the direction of the laser light is a significant factor in this measurement method. The result also revealed that, for the participant in the present study, the nose surface vibrated even when the vowel, not only just the nasal consonant was articulated.

\section{Discussion}

In this study, we proposed a novel method of measuring the vibration velocity patterns of skin surfaces during speech production. The obtained results demonstrated a clear contrast between the patterns for the vowel and the nasal consonant.

The scanning vibrometer is suitable for studying the speech sound radiation from the body surface; its frequency characteristics and the contribution of the sound radiated from the surfaces to the overall sound could be investigated. It is also interesting to determine the differences in the vibration patterns for various registers of singing.

The proposed method enables us to evaluate the speech of patients with cleft palate or velopharyngeal incompetence. The vibration pattern may be helpful as visual feedback of a speaking exercise for such patients. The vibration pattern may be easier to relate to their somesthesis than spectra of 
their speech sounds. This idea is also useful for singing exercises.

In conclusion, the proposed method allows noncontact and multipoint measurements of the vibration velocity of skin surfaces in a short time and will expand our knowledge of speech production. The next step that needs to be taken is to investigate the relationship between the vibration velocity pattern of skin surfaces and the formants and antiformants of speech sounds.

\section{Acknowledgments}

This study was supported by JSPS KAKENHI (21300071). The author wishes to thank Dr. Kazuhito Ito (National Institute of Advanced Industrial Science and Technology), Mr. Francois Bouteille, Mr. Ryo Ishiyama, Ms. Shoko Wakatsuki (Polytec Japan), and Professor Ken-ichi Sakakibara (Health Sciences University of Hokkaido) for their generous assistance and valuable advice.

\section{References}

[1] J. Sundberg, The Science of Singing Voice (Northern Illinois University Press, DeKalb, Ill., 1987).

[2] K. N. Stevens, Acoustic Phonetics (The MIT Press, Cambridge, London, 2000).

[3] I. Kirikae, T. Sato, H. Oshima and K. Nomoto, "Vibration of the body during phonation of vowels," Revue de Laryngologie, Otologie, Rhinologie, 85, 317-345 (1964).

[4] H. Suzuki, J. Dang and T. Nakai, "Measurement of sound and vibration at the lips, nostrils and pharynx wall in speech utterance and simulation of sound leakage from the oral cavity to the nasal cavity in non-nasal sounds," IEICE Trans., J74-A, 1705-1714 (1991).

[5] K. Toyoda and T. Fujinami, "An effective singing for musical expressions," Proc. 10th Int. Conf. Music Perception and Cognition, pp. 372-380 (2008).

[6] R. Pawluczyk and Z. Kraska, "Diffuse illumination in holographic double-aperture interferometry," Appl. Opt., 24, 30723078 (1985).

[7] Polytec web page, http://www.polytec.com/ 